\title{
TITLE:
}

\section{A study on the accuracy of surface charge measurement}

$\operatorname{AUTHOR}(\mathrm{S})$ :

Tatematsu, A; Hamada, S; Takuma, T; Morii, H

\section{CITATION:}

Tatematsu, A ... [et al]. A study on the accuracy of surface charge measurement. IEEE TRANSACTIONS ON DIELECTRICS AND ELECTRICAL INSULATION 2002, 9(3): 406-415

\section{ISSUE DATE:}

2002-06

URL:

http://hdl.handle.net/2433/39978

\section{RIGHT:}

(c)2002 IEEE. Personal use of this material is permitted. However, permission to reprint/republish this material for advertising or promotional purposes or for creating new collective works for resale or redistribution to servers or lists, or to reuse any copyrighted component of this work in other works must be obtained from the IEEE. 


\title{
A Study on the Accuracy of Surface Charge Measurement
}

\author{
Akiyoshi Tatematsu, Shoji Hamada, Tadasu Takuma \\ Department of Electrical Engineering \\ Kyoto University, Yoshida-honmachi \\ Sakyo-ku, Kyoto 606-8501, Japan \\ and Hiroshi Morii \\ Research and Development Department \\ The Kansai Electric Power Co. \\ 3-3-22, Nakanoshima, Kita-ku \\ Osaka 530-8270, Japan
}

\begin{abstract}
The measurement of accumulated surface charge for thick specimens requires multipoint probe outputs to establish the inverse calculation for the determination of an unknown charge distribution. Until now, studies on the various errors associated with the measurement have been conducted only for simplified arrangements mainly in axisymmetric geometry where the charged surface is parallel to the ground. We have numerically analyzed a model measurement set-up more comparable to practical conditions by a highly efficient surface charge method. We have studied the effect of probe position, the induction from charge existing not directly beneath the (probe) sensor and the difference in matrix components computed by two numerical methods. In particular, we have studied the accuracy of the reconstructed charge distributions by numerical simulations of the inverse calculation. It has been shown that the assumed measurement errors make much larger differences in the reconstructed charge distributions, although the influence depends considerably on the assumed charge distribution. Reducing the condition number of the matrix improves the accuracy of the inverse calculation for uniform and linearly changing charge distributions.
\end{abstract}

\section{INTRODUCTION}

$S^{\circ}$ OLID dielectrics are used as supports of stressed conductors in any $\mathrm{HV}$ insulation system. In compressed $\mathrm{SF}_{6}$ gas or vacuum insulation systems where high electric field is involved, charge accumulation on solid dielectrics may seriously influence surface discharge and insulation characteristics. Until recently, the reliable measurement of accumulated charge has been confined to thin specimens such as films and sheets. This is because in the atmosphere, surface charge makes serious problems only for thin specimens which hold their electrification for a long time on account of their large capacitance to ground. Little attention has been paid to thick specimens with small capacitance because of their short leakage time in the atmosphere. However, charge accumulation on the surface of solid insulating supports has now become a very significant problem under dry conditions such as in $\mathrm{SF}_{6}$ gas or vacuum for the enclosed insulation systems now widely used in the electric power industry.

In the measurement of accumulated charge for thin specimens mounted on a grounded conductor, a one- dimensional "uniform-field approximation" holds, and a probe output (induced charge on a sensor) corresponds uniquely to the accumulated charge (density) facing the probe. The measurement of accumulated surface charge is completely different for thick specimens such as solid supports (insulating spacers). It is necessary to form a matrix relationship between probe outputs and charge densities in the whole system. The surface charge distribution is calculated from the probe outputs obtained by a multipoint measurement through use of the inverse matrix. To evaluate coefficients in the matrix for three-dimensional arrangements, we must also apply numerical field calculation techniques.

As far as we know, the idea of the matrix relationship was reported for the first time in [1]. Several papers have since been published about procedures based on the multipoint measurement. Nevertheless, its principle is not fully understood. We consider this is mainly due to the complexity of the numerical method involved. One of us has published a paper on the principle of the surface charge measurement for thick specimens and, in particular, compared the mathematical expressions involved in the procedures adopted [2]. Until now, however, no quantitative 
measurements have been successfully made for practical spacers or insulating supports due to other difficulties in the multipoint measurement. Accumulated charge measurements were reported on $500 \mathrm{kV} \mathrm{dc}$ GIS [3-5]. But two of these papers adopted directly induced charge as a measured value at each spot, which is not based on the multipoint measurement, thus neglecting the effect of the surrounding charge $[3,4]$. One paper employed the multipoint measurement [5], but its principle is not correct as explained in [2].

We have improved the calculation accuracy of the matrix components for a simple model arrangement by comparing the values obtained through two numerical field calculation algorithms which should theoretically result in the same matrix $[6,7]$. The purpose of this paper is to analyze the accuracy in measuring accumulated charge distributions by the multipoint measurement and the inverse calculation for more practical conditions. We have numerically simulated the reconstruction or reproduction of the charge distributions by the probe outputs including measurement errors with the matrix coefficients computed by a field calculation. It is well known the inverse calculation easily becomes unstable. Thus the distributions reconstructed from the square inverse matrix often differ significantly from the assumed ones. By applying the singular value decomposition to the matrix and reducing the condition number of the matrix, we have reconstructed the assumed distributions more correctly in some cases.

\section{MODEL ARRANGEMENT}

In our previous papers, we have analyzed a model arrangement which simulates the measurement performed at the Central Research Institute of Electric Power Industry (CRIEPI) [8]. The measurement at CRIEPI was for the accumulated charge on the surface of a dielectric block. The block had a square upper surface of $2.5 \times 2.5$ $\mathrm{cm}^{2}$ and a height of $1 \mathrm{~cm}$. It was divided into 25 smaller blocks, with an upper area of $0.5 \times 0.5 \mathrm{~cm}^{2}$. The upper surface of each smaller block was artificially charged to a constant value. The charge densities directly measured by the Faraday cage technique were compared with those calculated from the probe outputs. We have already reported that the values of the matrix components calculated through two algorithms agree well with each other for the model arrangement in which a probe is simply simulated by a square plate (sensor) without a guard electrode $[6,7]$.

Figure 1 shows the model arrangement for the analysis in this paper. The solid dielectric is not a block as in the previous papers but a cube with a side of $2.5 \mathrm{~cm}$, and a relative permittivity of 4 . Although various shapes are used in practical insulating supports, we have adopted the cube to avoid the inclusion of too many parameters. The solid lies on a grounded plane $(h=0 \mathrm{~cm})$, or it is situated $3 \mathrm{~cm}$ above the ground. These two situations are chosen to investigate the effect of the ground. This is because al-

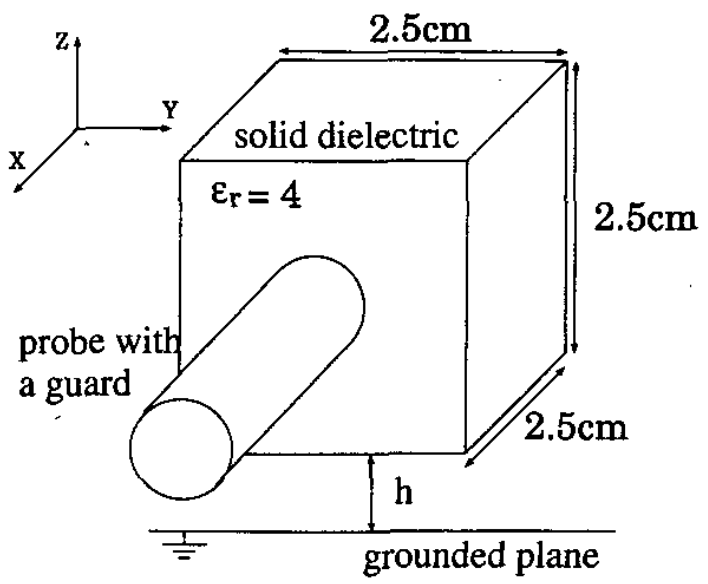

Figure 1. Model arrangement.

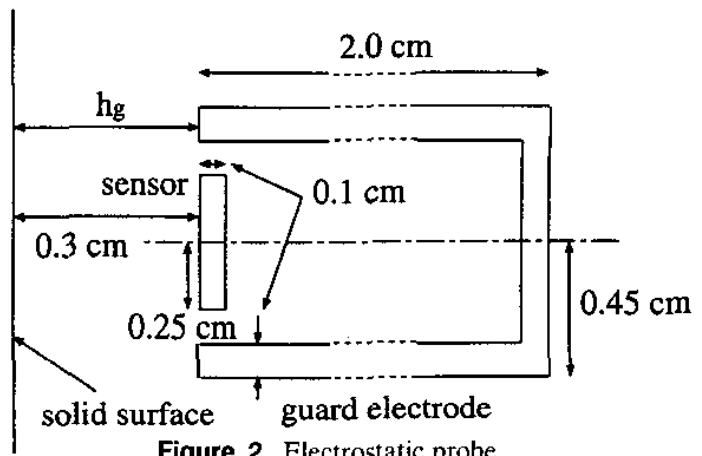

Figure 2. Electrostatic probe.

though a spacer usually lies on a grounded electrode, it is inserted between two grounded electrodes during charge measurement in a co-cylindrical arrangement.

Figure 2 represents the cylindrical electrostatic probe consisting of a sensor (sensing plate) surrounded by a grounded guard electrode. The sensor is a disc $0.5 \mathrm{~cm}$ in diameter and $0.1 \mathrm{~cm}$ in thickness. The guard electrode is a cylinder of inner radius $0.35 \mathrm{~cm}$ and outer radius $0.45 \mathrm{~cm}$ with a length of $2 \mathrm{~cm}$. In the measurement for thin specimens, a guard electrode is inevitably necessary to ensure the uniform field condition near the sensor, and thus commercial probes are always fitted with a guard electrode. However, in the multipoint measurement based on the matrix relationship, the guard is not always considered indispensable. As the previous calculation was performed for a simplified probe without a guard, we have also analyzed the probe output without a guard in some cases of this study. In the actual measurement, the sensor is usually grounded through a measuring capacitance. If the capacitance is sufficiently large, the sensor is at a virtual ground. Therefore, the potential of the sensor is set to zero in this paper. The sensor of the electrostatic probe is located at a distance $h_{g}$ from the side surface of the dielectric. This distance is kept at $0.3 \mathrm{~cm}$ in the present calculation up to section 5 . 


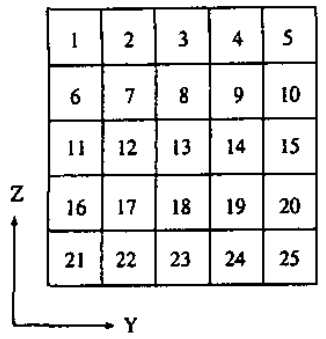

(a)

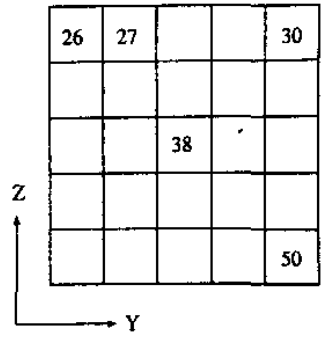

(b)
Figure 3. Labeling of subareas of the dielectric. (a) side facing probe; (b) rear side.

Although actual charge densities change continuously on the surface, we approximate them as discrete values for the later inverse calculation. We divide the solid (cube) surface facing the probe and the rear (opposite side) surface as possible charged areas, respectively, into 25 subareas as shown in Figure 3. In this paper, we do not consider the charging in the other side surfaces. Each subarea has an area of $0.5 \times 0.5 \mathrm{~cm}^{2}=0.25 \mathrm{~cm}^{2}$, while the area of the sensor is $0.196 \mathrm{~cm}^{2}$. Note that this subdivision of the surfaces is different from that in the numerical field calculation where the mesh must be finer to attain enough accuracy. Examples of the subdivision for the field calculation are shown later in Figure 6.

The charge density $\sigma(j)$ on subarea $j$ is assumed constant and the position of the probe is designated as $i$. The probe output $W_{p}(i)$, that is, the charge induced on the sensor at position $i$ by $\sigma(j)$ is expressed as

$$
W_{p}(i)=A(i, j) \sigma(j)
$$

where the coefficient $A(i, j)$ is obtained by the numerical field calculation. Two numerical field calculation atgorithms have been employed to evaluate the coefficient, namely, the direct method (called the matrix method I in [2] and [6]) and the $\lambda$-function method $[9,10]$.

For field calculation in the three-dimensional arrangement, we have applied the surface charge (simulation) method highly developed in our laboratory, which is briefly explained in the Appendix. Figure 4 shows the mesh used for the sensor of the probe in the field calculation. Figure 5 represents the mesh for the guard electrode. Although (a)

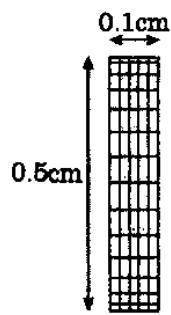

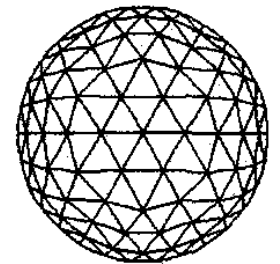

(b)
Figure 4. Computational mesh for the sensor. (a) side; (b) upper and lower faces.

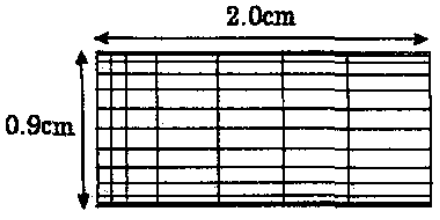

(a)



(b)

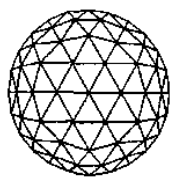

(c)

Figure 5. Computational mesh for the guard electrode. (a) outer side; (b) sensor side-end; (c) rear end.

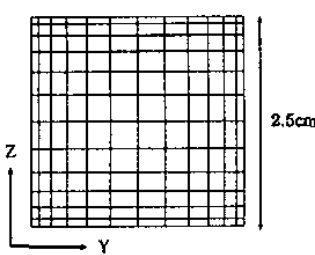

(a)



(b)

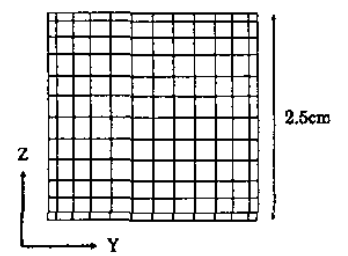

(c)

Figure 6. Examples of computational mesh for the solid surface. (a) mesh for Figure 7a; (b) mesh for Figure $7 \mathrm{~b}$ or $7 \mathrm{c}$; (c) mesh for computing matrix components.

Figure 5 shows only the outside surface, the inside surface of the guard is also similarly subdivided. Figure 6 represents three examples of the meshes for the solid surface of $2.5 \times 2.5 \mathrm{~cm}^{2}$ facing the probe. The mesh pattern for the other surfaces of the cube not facing the probe is similar to that of Figure $6 \mathrm{a}$, but the finer mesh is only near the edge closer to the probe because the mesh pattern has little influence on the accuracy of the field calculation. The mesh patterns shown in Figure 6 are merely examples. We have applied different mesh patterns for the solid in accordance with the assumed charge distributions.

\section{PROBE OUTPUT 3.1 EFFECT OF MEASUREMENT POSITION}

Table 1 summarizes the probe outputs at several probe positions, when the charge density is constant on the whole surface of one side of the solid. The outputs are numeri- 
Table 1. Calculated probe output versus measurement position, (a) $h=3 \mathrm{~cm}$; (b) $h=0 \mathrm{~cm}$.

\begin{tabular}{lcc} 
(a) $h=3 \mathrm{~cm}$ & & \\
& & \\
position $i$ & output $W_{p}(i)[\mathrm{pC}]$ & \\
\cline { 2 - 3 } & without the guard & with the guard \\
\hline 1 & 2.82 & 0.76 \\
13 & 3.52 & 0.97 \\
(b) $h=0 \mathrm{~cm}$ & 3.72 & 1.01 \\
\hline & & \\
position $i$ & without the guard & with the guard \\
\hline 1 & 1.69 & 0.54 \\
7 & 2.14 & 0.69 \\
13 & 2.08 & 0.69 \\
19 & 1.57 & 0.56 \\
\hline
\end{tabular}

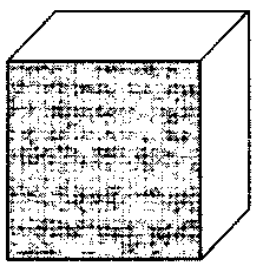

(a)



(c)

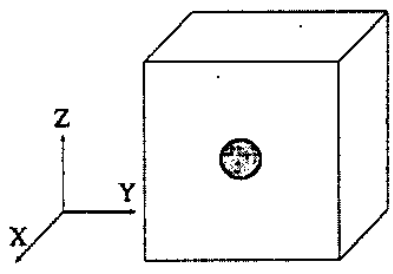

(b)

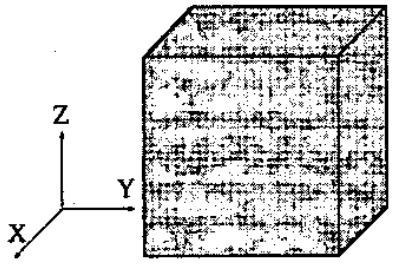

(d)
Figure 7. Four test charge distributions.

cal values calculated by superposing the electrostatic induction on the probe sensor based on equation (1) as the effect of the charge densities on the subareas. In Table 1, position $i$ corresponds to a subarea number of Figure 3a. The charge density is $4 \mathrm{pC} / \mathrm{cm}^{2}$ on the whole surface of the side facing the probe as shown in Figure 7a. The charge on an area directly beneath the sensor is $0.78 \mathrm{pC}$ unless the probe is at the edge of a cube side.

As already mentioned, the calculation gives an almost constant probe output for thin specimens irrespective of probe position, when the solid surface is charged uniformly at a constant density [2,8]. On the other hand, the output for thick samples varies significantly with position as Table 1 shows. In the case of $h=3 \mathrm{~cm}$, the highest output appears at the center of $i=13$. The output decreases by about $25 \%$ at the edge zone of $i=1$ both for the sensor alone (without a guard electrode) and for the sensor with the guard. When the solid lies on the ground $(h=0 \mathrm{~cm}$ ), the output at $i=1$ decreases by about $20 \%$ in comparison with that at $i=13$, but the highest output appears at the higher location $i=7$. This is due to the charge of opposite polarity induced on the ground. In any calcu-
Table 2. Calc llated probe output for the charge distributions shown in Figure 7 (probe position $i=13$ ). (a) $h=3 \mathrm{~cm}$; (b) $h=0 \mathrm{~cm}$.

\begin{tabular}{lcc} 
(a) $h=3 \mathrm{~cm}$ & & \\
& & \\
charging & output $W_{p}(i)[\mathrm{pC}]$ & \\
\cline { 2 - 3 } without the guard & with the guard \\
\hline (a) & 3.72 & 1.01 \\
(b) & 0.20 & 0.10 \\
(c) & 3.51 & 0.91 \\
(d) & 14.4 & 3.67 \\
\hline (b) $h=0 \mathrm{~cm}$ & & \\
\hline & & \\
charging & output $W_{p}(i)[\mathrm{pC}]$ & \\
\hline (a) & without the guard & with the guard \\
(b) & 2.08 & 0.69 \\
(c) & 0.16 & 0.09 \\
(d) & 1.92 & 0.59 \\
\hline
\end{tabular}

lated case, the probe output without the guard is much higher than the corresponding charge of $0.78 \mathrm{pC}$ directly beneath the sensor. The guard electrode considerably reduces the output, but the output still do not coincide with each other among various probe positions.

The reason why the probe output varies at each position is that the output depends not only on the charge directly beneath the sensoribut also on the entire charge distribution. The effect of the surrounding charge is demonstrated in more detail in the following results.

\subsection{EFFECT OF CHARGE ON SURROUNDING SURFACES}

Table 2 compares the probe outputs for four cases of charge accumulation on the solid when the probe is situated facing the center of the solid side $(i=13)$ at a distance of $0.3 \mathrm{~cm}$ from the solid surface. Figure 7 shows the four charge distributions as follows:

(a) charge on the whole surface of the side nearest the probe.

(b) charge on a circular area of $0.5 \mathrm{~cm}$ in diameter at the center.

(c) charge on the whole surface of one side except the area of (b).

(d) charge on the whole surface of all six sides.

In case (b), the charge exists only on an area directly beneath the sensor. The charge density is constant at 4 $\mathrm{pC} / \mathrm{cm}^{2}$ in all cases. It is self-evident that the probe output for case (a) should be equal to the sum of the corresponding values for cases (b) and (c). Comparing cases (a) and (b) reveals that the greater part of the output is induced by the charge not directly beneath the sensor. When the solid lies $3 \mathrm{~cm}$ above the ground, the ratio of these outputs is only about $5 \%$ for the measurement without the guard and $10 \%$ with the guard. In the case of the solid on the ground $(h=0 \mathrm{~cm})$, the corresponding values are about $8 \%$ without the guard and $13 \%$ with the guard. If the whole surface of the solid is charged, case (d), the contribution of the charge directly beneath the sensor fur- 
ther diminishes to only $3-6 \%$ even in the measurement with a guard electrode.

This effect of the surrounding charge has already been reported for the simplified arrangement in axisymmetric geometry where the height of the block is considerably smaller than the side length and the charged surface is in parallel with the ground $[8,10]$. The present study analyzes the effect for the charging on the side surface of the solid cube dielectric, which is much more comparable to a practical insulating support. In this arrangement, the effect of the surrounding charge is much larger than in the flat slab dielectric.

\section{MULTIPOINT MEASUREMENT}

As explained above, a surface charge distribution is evaluated from the multipoint measurement and the inverse calculation based on the matrix relationship as shown in equation (2). Since the inverse calculation is apt to become unstable, even a slight error may bring about a large difference in the calculated charge distribution. Consequently, it is very important to calculate coefficients (matrix components) as accurately as possible. Two numerical calculation algorithms have been employed to evaluate the components in the matrix, namely the direct method (called the matrix method $\mathrm{I}$ in [2] and [6]) and the $\lambda$-function method $[9,10]$. We briefly explain these methods below. Theoretically these two methods should result in the same matrix. In practice, however, these differ owing to numerical errors. We have previously reported the comparison of the matrix components for the simple geometry computed by the two algorithms $[6,7]$.

\subsection{DIRECT METHOD (MATRIX METHOD I) [2]}

This method directly combines accumulated charge on a surface of a solid dielectric with charge on a probe sensor induced by the surface charge. The probe output is expressed as a linear combination of the effects of all the charge in the system. If all the conductors in the system are grounded, then the probe output $W_{p}(i)$ at position $i$ is

$$
W_{p}(i)=\sum_{j=1}^{N} A(i, j) \sigma(j)
$$

where $\sigma(j)$ is a charge density at a subarea $j$ when the charged surface area is divided into $N$ subareas, each of which has a uniform charge density $\sigma(j)$. We can compute the coefficient $A(i, j)$ by applying a numerical field calculation method corresponding to the case of unit charge density on the subarea $j$.

\section{$4.2 \lambda$-FUNCTION METHOD $[9,10]$}

This method starts from the following relationship between accumulated charge density $d Q$ on an elementary surface area $d S$ of the insulating specimien and the charge $d q$ which $d Q$ induces electrostatically on the sensor.

$$
d q=-\lambda d Q=-\lambda \sigma d S
$$

Hence the total induced charge on the sensor is

$$
q=-\iint_{S_{0}} \lambda \sigma d S
$$

The proportion coefficient is called the " $\lambda$-function", a dimensionless variable depending only on the location of $d Q$ for the given arrangement.

This method differs from the direct method in that the $\lambda$-function giving the matrix coefficients is calculated by

$$
\operatorname{div}(\epsilon \operatorname{grad} \lambda)=0
$$

with the boundary conditions $\lambda=1$ on the sensor plate, $\lambda=0$ on all the other electrode surfaces, and the continuity of the normal derivative of $\lambda$ on the dielectric interface. Equation (5) is simply Laplace's equation for potential $\lambda$, and in brief the $\lambda$-function corresponds to a function representing the proportion of the resulting potential on the dielectric surface to the potential of the sensor. See [10] for details.

For a subarea $j$ with a constant $\sigma(j)$, equation (4) can be rewritten as

$$
q=-\sigma(j) \iint_{S(j)} \lambda d S
$$

where $S(j)$ designates the area of a subarea $j$. By discretizing the integral in equation (6), it can be shown that the total probe output at location $i$ can also be expressed as

$$
W_{p}(i)=\sum_{j=1}^{N} A(i, j) \sigma(j)
$$

where $A(i, j)$ is now given by

$$
A(i, j)=\iint_{S(j)} \lambda d S
$$

\subsection{MATRIX COMPONENTS BY THE TWO METHODS}

Table 3 compares several components calculated by the two methods for the arrangement of Figure 1 with $h=3$ $\mathrm{cm}$. Since the true value of the component is unknown, the percentage difference $D$ has been calculated as follows,

$$
\begin{aligned}
A_{\text {ave }} & =\left(A_{D}+A_{\lambda}\right) / 2 \\
D & =\left|A_{D}-A_{\lambda}\right| / A_{a v e} \times 100
\end{aligned}
$$

Table 3. Comparison of matrix components $(h=3 \mathrm{~cm})$.

\begin{tabular}{lccc}
\hline matrix component & direct & $\lambda$-function & difference [\%] \\
\hline$A(1,1)$ & 0.14588 & 0.14698 & 0.75 \\
$A(13,13)$ & 0.12269 & 0.12289 & 0.16 \\
$A(1,25)$ & 0.01294 & 0.01317 & 1.76 \\
$A(1,26)$ & 0.01673 & 0.01687 & 0.83 \\
$A(13,38)$ & 0.01925 & 0.01930 & 0.26 \\
$A(1,50)$ & 0.01145 & 0.01166 & 1.82 \\
\hline
\end{tabular}


where $A_{D}$ and $A_{\lambda}$ are the coefficient computed by the direct method and the $\lambda$-function method, respectively. In all the cases, the differences are below $2 \%$. It must be noted that the differences of $A(1,1)$ and $A(13,13)$ are $0.75 \%$ and $0.16 \%$, respectively. These are diagonal matrix components, usually larger and more important than the other elements in the respective row and column. On the other hand, the difference of $A(1,25)$ is roughly twice as large as that of $A(1,1)$. However, since the value itself is only one-tenth of $A(1,1)$, the difference has less influence on the inverse calculation than that of the diagonal component. Three matrix components $A(1,26), A(13,38)$ and $A(1,50)$ represent the coefficients between the probe output and the charge on the rear surface of the solid, as shown in Figure 3b. These components by the two methods are also close to each other with the difference ranging from 0.2 to $1.8 \%$.

In the previous comparison for the simple geometry, the difference of diagonal components, $A(1,1)$ and $A(13,13)$, were smaller, $0.15 \%$ in minimum, than for present case. The larger difference is due to the more complicated arrangement in the present casc and the very fine subdivision in the previous calculation. However, the differences below $0.8 \%$ for the diagonal components are considered sufficiently small in the following inverse calculations. The effect of the difference in matrix components is further discussed in Section 5.

\section{INVERSE CALCULATION}

\subsection{EFFECT OF THE GUARD ELECTRODE POSITION}

As already explained, the guard electrode is not considered indispensable to ensure the quasi-uniform condition in the multipoint measurement. Therefore, we have examined the effect of the position of the guard electrode by changing $h_{g}$ shown in Figure 2 to smaller than $0.3 \mathrm{~cm}$, but with the sensor still at $0.3 \mathrm{~cm}$. This means that the guard projected beyond the face of the sensor. When the guard electrode approaches the solid surface too closely, there is the possibility of a discharge, leading to incorrect readings. For this reason, the distance between the guard and the surface was limited to $0.2 \mathrm{~cm}$ at the closest.

It is well known that a matrix is better conditioned when its diagonal component has a value larger than the other components in the corresponding row or column. A better-conditioned matrix more effectively suppresses the enlargement of the errors involved in the inverse calculation, as explained later. Table 4 shows the values of two matrix

Table 4. Effect of the guard elcctrode position $(h=3 \mathrm{~cm})$.

\begin{tabular}{cccc}
\hline$h_{g}[\mathrm{~cm}]$ & $A(13,13)$ & $A(13,14)$ & $A(13,14) / A(13,13)$ \\
\hline 0.3 & 0.12269 & 0.06299 & 0.52 \\
0.25 & 0.10481 & 0.04859 & 0.47 \\
0.2 & 0.08999 & 0.03709 & 0.41 \\
\hline
\end{tabular}

components, $A(13,13)$ and its neighbor $A(13,14)$, and the ratio of $A(13,14)$ to $A(13,13)$ for each guard position. These values were calculated by the direct method. The coefficients calculated by the $\lambda$-function method differed less than $0.7 \%$. Table 4 indicates that the ratio decreases with decreasing $h_{g}$, becoming smallest for $h_{g}=0.2 \mathrm{~cm}$, although the difference is not very large. Consequently, in the later calculations (Section 5) the guard electrode is situated at the distance of $0.2 \mathrm{~cm}$ from the solid surface while the sensor is recessed by $0.1 \mathrm{~cm}$.

\subsection{REPRODUCED CHARGE DISTRIBUTION}

As described above, the numerical values of the matrix coefficients computed by the direct method are not identical to the ones by the $\lambda$-function method on account of numerical errors. Thus the two results obtained by the inverse calculation will differ. The differences of the matrix components may give rise to more significant errors in the reproduced charge distribution through the inverse calculation than the values themselves. By numerically simulating the procedure, we have analyzed the charge distribution reconstructed or reproduced from the multipoint measurement and the inverse calculation with the components calculated by the two methods. The following four charge distributions have been investigated:

(i) The whole surface of one side is uniformly charged at a constant density of $4 \mathrm{pC} / \mathrm{cm}^{2}$ as shown in Figure $7 \mathrm{a}$.

(ii) Only the subarea $j=13$ (of $0.5 \times 0.5 \mathrm{~cm}^{2}$ ) in Figure $3 \mathrm{a}$ is charged uniformly at $4 \mathrm{pC} / \mathrm{cm}^{2}$.

(iii) The subareas $j=12$ and 14 are charged uniformly at $4 \mathrm{pC} / \mathrm{cm}^{2}$ and $-4 \mathrm{pC} / \mathrm{cm}^{2}$, respectively.

(iv) The whole of the front side is charged at a density which changes smoothly (explained in detail later).

The solid is situated at $3 \mathrm{~cm}$ above the ground $(h=3$ $\mathrm{cm})$. Twenty-five $(=5 \times 5)$ probe outputs are the values as computed by the surface charge method. The differences between the diagonal components $A(i, i)(i=1-25)$ of the two matrices calculated by the two methods are $0.38-0.65 \%$.

Table 5 shows the charge distribution thus obtained by the inverse calculation for case (i). The layout of the table matches the layout of Figure $3 \mathrm{a}$. There is a charge of $1 \mathrm{pC}$ $\left(=4 \mathrm{pC} / \mathrm{cm}^{2} \times 0.25 \mathrm{~cm}^{2}\right)$ in each subarea. The charge distribution reproduced from equation (2) with the matrix components by the direct method has given reasonably accurate values where the largest error is only $1.3 \%$. On the other hand, the result derived from the matrix by the $\lambda$ function method is a little worse with a maximum error of about $3 \%$.

Table 6 presents in the same way the distribution calculated with the matrix inverse by the two methods for case (ii), which has local charge accumulation only at the center subarea $j=13$. The direct method reproduces the assumed distribution correctly, with no error in the subarea 
Table 5. Charge distribution reproduced for Figure $7 \mathrm{a}(h=3 \mathrm{~cm})$. (a) direct method (in pC); (b) $\lambda$-function method (in pC).

\begin{tabular}{cllll}
\hline (a) direct method & & & & \\
\hline 0.994 & 1.00 & 0.933 & 1.00 & 0.994 \\
1.00 & 1.01 & 0.987 & 1.01 & 1.00 \\
0.993 & 0.987 & 1.01 & 0.987 & 0.993 \\
1.00 & 1.01 & 0.987 & 1.01 & 1.00 \\
0.994 & 1.00 & 0.993 & 1.00 & 0.994 \\
\hline (b) $\lambda$-function method & & & & \\
\hline 0.968 & 0.994 & 0.968 & 0.994 & 0.968 \\
0.994 & 1.03 & 0.992 & 1.03 & 0.994 \\
0.968 & 0.992 & 0.994 & 0.992 & 0.968 \\
0.994 & 1.03 & 0.992 & 1.03 & 0.994 \\
0.968 & 0.994 & 0.968 & 0.994 & 0.968 \\
\hline
\end{tabular}

Table 6. Charge distribution reproduced for charge on subarea $j=$ $13(h=3 \mathrm{~cm})$. (a) direct method (in pC); (b) $\lambda$-function method (in pC).

\begin{tabular}{crcrr}
\hline (a) direct method & & & & \\
\hline $5.1 \mathrm{E}-5$ & $-1.8 \mathrm{E}-5$ & $1.0 \mathrm{E}-4$ & $-1.8 \mathrm{E}-5$ & $5.1 \mathrm{E}-5$ \\
$-1.9 \mathrm{E}-5$ & $2.1 \mathrm{E}-4$ & $-3.6 \mathrm{E}-4$ & $2.1 \mathrm{E}-4$ & $-1.9 \mathrm{E}-5$ \\
$1.0 \mathrm{E}-4$ & $-3.6 \mathrm{E}-4$ & 1.00 & $-3.6 \mathrm{E}-4$ & $1.0 \mathrm{E}-4$ \\
$-1.9 \mathrm{E}-5$ & $2.1 \mathrm{E}-4$ & $-3.6 \mathrm{E}-4$ & $2.1 \mathrm{E}-4$ & $-1.9 \mathrm{E}-5$ \\
$5.1 \mathrm{E}-5$ & $-1.9 \mathrm{E}-5$ & $1.0 \mathrm{E}-4$ & $-1.9 \mathrm{E}-5$ & $5.1 \mathrm{E}-5$ \\
\hline (b) $\lambda$-function method & & & & \\
\hline$-4.2 \mathrm{E}-4$ & $4.8 \mathrm{E}-4$ & $-1.5 \mathrm{E}-3$ & $4.8 \mathrm{E}-4$ & $-4.2 \mathrm{E}-4$ \\
$4.9 \mathrm{E}-4$ & $-3.5 \mathrm{E}-3$ & $6.1 \mathrm{E}-3$ & $-3.5 \mathrm{E}-3$ & $4.9 \mathrm{E}-4$ \\
$-1.5 \mathrm{E}-3$ & $6.1 \mathrm{E}-3$ & 0.986 & $6.1 \mathrm{E}-3$ & $-1.5 \mathrm{E}-3$ \\
$4.9 \mathrm{E}-4$ & $-3.5 \mathrm{E}-3$ & $6.1 \mathrm{E}-3$ & $-3.5 \mathrm{E}-3$ & $4.9 \mathrm{E}-4$ \\
$-4.1 \mathrm{E}-4$ & $4.9 \mathrm{E}-4$ & $-1.5 \mathrm{E}-3$ & $4.9 \mathrm{E}-4$ & $-4.1 \mathrm{E}-4$ \\
\hline
\end{tabular}

of $j=13$ and very small values in the other subareas. However, the $\lambda$-function method gives a value lower by $1.4 \%$ in the subarea $j=13$, and larger values by one order of magnitude in the other subareas.

The reproduced distribution for case (iii), in which both positive and negative charges exist, is represented in Table 7. The distribution obtained by the direct method has the values in subareas 12 and 14 identical with the original ones, while the $\lambda$-function method gives a difference of $1.3 \%$.

In case (iv), the front surface of the cube has a charge density which changes continuously as a linear function of

Table 7. Charge distribution reproduced for positive charge on subarea $j=12$ and negative on $j=14(h=3 \mathrm{~cm})$. (a) direct method (in pC); (b) $\lambda$-function method (in pC).

\begin{tabular}{cccrr}
\hline (a) direct method & & & & \\
\hline$-3.9 \mathrm{E}-6$ & $6.4 \mathrm{E}-5$ & 0.0 & $-6.4 \mathrm{E}-5$ & $3.9 \mathrm{E}-6$ \\
$1.2 \mathrm{E}-4$ & $-2.2 \mathrm{E}-4$ & 0.0 & $2.2 \mathrm{E}-4$ & $-1.2 \mathrm{E}-4$ \\
$-1.4 \mathrm{E}-4$ & 1.00 & 0.0 & -1.00 & $1.4 \mathrm{E}-4$ \\
$1.2 \mathrm{E}-4$ & $-2.2 \mathrm{E}-4$ & 0.0 & $2.2 \mathrm{E}-4$ & $-1.2 \mathrm{E}-4$ \\
$-3.8 \mathrm{E}-6$ & $6.4 \mathrm{E}-5$ & 0.0 & $-6.4 \mathrm{E}-5$ & $3.8 \mathrm{E}-6$ \\
\hline (b) $\lambda$-function method & & & & \\
\hline $4.4 \mathrm{E}-4$ & $-8.8-4$ & $2.4 \mathrm{E}-8$ & $8.8 \mathrm{E}-4$ & $-4.4 \mathrm{E}-4$ \\
$-4.3 \mathrm{E}-3$ & $5.8 \mathrm{E}-3$ & $-2.9 \mathrm{E}-8$ & $-5.8 \mathrm{E}-3$ & $4.3 \mathrm{E}-3$ \\
$5.2 \mathrm{E}-3$ & 0.987 & $5.7 \mathrm{E}-8$ & -0.987 & $-5.2 \mathrm{E}-3$ \\
$-4.3 \mathrm{E}-3$ & $5.8 \mathrm{E}-3$ & $2.0 \mathrm{E}-8$ & $-5.8 \mathrm{E}-3$ & $4.3 \mathrm{E}-3$ \\
$4.4 \mathrm{E}-4$ & $-8.8 \mathrm{E}-4$ & $-8.2 \mathrm{E}-9$ & $8.8 \mathrm{E}-4$ & $-4.4 \mathrm{E}-4$ \\
\hline
\end{tabular}

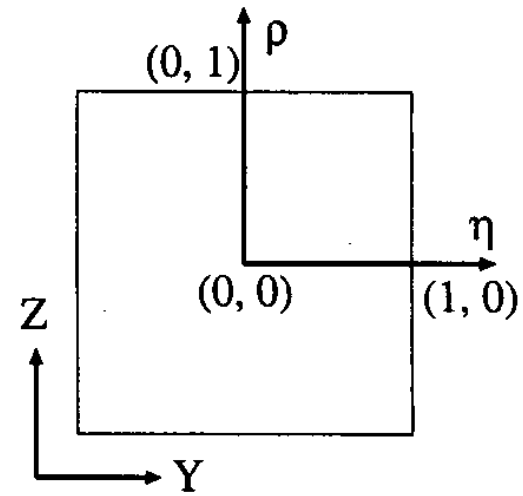

Figure 8. Coordinates for the front side of the cube.



(a)



(b)
Figure 9. Charge distribution (iv). (a) charge density in $\mathrm{pC} / \mathrm{cm}^{2}$; (b) charge distribution integrated over each subarea in $\mathrm{pC}$.

the coordinates. We define coordinates $\eta$ and $\rho(-1 \leq \eta$, $\rho \leq 1)$ for the side of the solid facing the probe as shown in Figure 8. Then, the charge density on the surface is expressed as

$$
\sigma=(1-\eta)(1-\rho) \sigma_{0} / 4
$$

where $\sigma_{0}=4 \mathrm{pC} / \mathrm{cm}^{2}$. Figure $9 \mathrm{a}$ shows the charge density distribution corresponding to equation (10), while Figure $9 \mathrm{~b}$ shows the result if the same charge density is distributed uniformly on each subarea, corresponding to Table 8a.

Numerical values are given in Table $8 \mathrm{a}$. These values have been computed by the numerical integration of $\sigma$ in each subarea. Table $8 \mathrm{~b}$ shows the result inversely calculated with the matrix by the direct method by considering a constant charge density in each subarea as in equation (2). Similar values for the $\lambda$-function method are given in Table $8 \mathrm{c}$. Although the reproduced distribution consists of discrete values in 25 subareas, the results in Tables $8 \mathrm{~b}$ and $8 \mathrm{c}$ agree well with those in Table $8 \mathrm{a}$ with the differences below $4 \%$ at the subareas where the accumulated charge is larger than $0.1 \mathrm{pC}$. Comparing the results by the two methods, the $\lambda$-function method leads to smaller differences than the direct method in most subareas charged to a value larger than $0.1 \mathrm{pC}$. In the other subareas, the differences of the direct method are smaller than those of the $\lambda$-function method, but the values of charge them- 
IEEE Transactions on Dielectrics and Electrical Insulation

Table 8. Assumed and reproduced charge distributions for Figure 9a $(h=3 \mathrm{~cm}$ ). (a) assumed distribution (in $\mathrm{pC}$ ); (b) direct method (in pC); (c) $\lambda$-function method (in pC).

\begin{tabular}{ccccc}
\hline (a) assumed distribution [pC] & & & & \\
\hline 0.810 & 0.630 & 0.450 & 0.270 & 0.090 \\
0.630 & 0.490 & 0.350 & 0.210 & 0.070 \\
0.450 & 0.350 & 0.250 & 0.150 & 0.050 \\
0.270 & 0.210 & 0.150 & 0.090 & 0.030 \\
0.090 & 0.070 & 0.050 & 0.030 & 0.010 \\
\hline (b) direct method [pC] & & & & \\
\hline 0.837 & 0.641 & 0.456 & 0.279 & 0.077 \\
0.641 & 0.490 & 0.345 & 0.214 & 0.059 \\
0.456 & 0.345 & 0.252 & 0.148 & 0.041 \\
0.279 & 0.214 & 0.148 & 0.095 & 0.026 \\
0.077 & 0.059 & 0.041 & 0.026 & 0.006 \\
\hline (c) $\lambda$-function method [pC] & & & & \\
\hline 0.822 & 0.636 & 0.447 & 0.276 & 0.073 \\
0.636 & 0.494 & 0.347 & 0.217 & 0.059 \\
0.447 & 0.347 & 0.249 & 0.149 & 0.038 \\
0.276 & 0.217 & 0.149 & 0.097 & 0.026 \\
0.073 & 0.059 & 0.038 & 0.026 & 0.004 \\
\hline & & & &
\end{tabular}

selves are very small in these subareas. As a whole, the $\lambda$-function method seems to reproduce the linearly distributed charge density more correctly.

In cases (i) to (iii), the assumed distributions are derived more accurately by the direct method. In the measurement of practical supports, however, the charge distribution is neither constant nor discrete, but changes continuously on the surface. Therefore, the result for Figure 9a suggests that the $\lambda$-function method may be more suitable for actual measurements, though more examination is necessary.

\subsection{EFFECT OF A MEASUREMENT ERROR OF PROBE OUTPUT}

The probe output in practice will"contain measurement errors, which, as already mentioned, may often bring larger errors in the inverse calculation. We have examined this point for a few typical cases given in Section 5.2 by introducing artificial errors in some of the probe outputs. Table 9 shows the result of the inverse calculation with the in-

Table 9. Effect of error in probe output on reproduced charge distribution for case (i). (a) $-5 \%$ error at position $i=1$; (b) $-5 \%$ error at position $i=13$.

\begin{tabular}{ccccc}
\hline (a) $-5 \%$ error at position $i=1$ & & & & \\
\hline 0.69 & 1.11 & 0.98 & 1.01 & 1.00 \\
1.11 & 1.01 & 0.99 & 1.02 & 1.01 \\
0.98 & 0.99 & 1.01 & 0.99 & 0.99 \\
1.01 & 1.02 & 0.99 & 1.01 & 1.01 \\
1.00 & 1.01 & 0.99 & 1.01 & 1.00 \\
\hline (b) $-5 \%$ error at position $i=13$ & & & & \\
\hline 1.00 & 1.01 & 0.98 & 1.01 & 1.00 \\
1.01 & 1.00 & 1.12 & 1.00 & 1.01 \\
0.98 & 1.12 & 0.46 & 1.12 & 0.98 \\
1.01 & 1.00 & 1.12 & 1.00 & 1.01 \\
1.00 & 1.01 & 0.98 & 1.01 & 1.00 \\
\hline
\end{tabular}

Vol. 9, No. 3, June 2002

Table 10. Effect of error in probe output at position ${ }^{-} i=13$ on reproducied charge distribution for case (ii) of charging on $j=13$.

$\begin{array}{rrrrr}4.9 \mathrm{E}-4 & 8.5 \mathrm{E}-4 & -2.4 \mathrm{E}-3 & 8.5 \mathrm{E}-4 & 4.9 \mathrm{E}-4 \\ 8.5 \mathrm{E}-4 & -1.1 \mathrm{E}-3 & 2.0 \mathrm{E}-2 & -1.1 \mathrm{E}-3 & 8.5 \mathrm{E}-4 \\ -2.4 \mathrm{E}-3 & 2.0 \mathrm{E}-2 & 0.917 & 2.0 \mathrm{E}-2 & -2.4 \mathrm{E}-3 \\ 8.5 \mathrm{E}-4 & -1.1 \mathrm{E}-3 & 2.0 \mathrm{E}-2 & -1.1 \mathrm{E}-3 & 8.5 \mathrm{E}-4 \\ 4.6 \mathrm{E}-4 & 8.5 \mathrm{E}-4 & -2.4 \mathrm{E}-3 & 8.5 \mathrm{E}-4 & 4.6 \mathrm{E}-4\end{array}$

Table 11. Effect of error in probe output at position $i=12$ on reproduced charge distribution for case (iii) of positive charge on subarea $j=12$ and negative on $j=14$.

\begin{tabular}{rcrcc}
$8.1 \mathrm{E}-4$ & $-2.0 \mathrm{E}-3$ & $7.0 \mathrm{E}-4$ & $1.9 \mathrm{E}-4$ & $2.5 \mathrm{E}-4$ \\
$-7.5 \mathrm{E}-4$ & $1.7 \mathrm{E}-2$ & $-1.1 \mathrm{E}-3$ & $8.2 \mathrm{E}-4$ & $7.9 \mathrm{E}-5$ \\
$1.5 \mathrm{E}-2$ & 0.933 & $1.7 \mathrm{E}-2$ & -1.00 & $8.5 \mathrm{E}-4$ \\
$-7.5 \mathrm{E}-4$ & $1.7 \mathrm{E}-2$ & $-1.1 \mathrm{E}-3$ & $8.2 \mathrm{E}-4$ & $7.7 \mathrm{E}-5$ \\
$8.1 \mathrm{E}-4$ & $-2.0 \mathrm{E}-3$ & $7.0 \mathrm{E}-4$ & $1.8 \mathrm{E}-4$ & $2.4 \mathrm{E}-4$ \\
\hline
\end{tabular}

Table 12. Effect of error in probe output on reproduced charge distribution for case (iv). (a) $-5 \%$ error at position $i=1$; (b) $-5 \%$ error at position $i=13$.

\begin{tabular}{ccccc}
\hline (a) $-5 \%$ error at position $i=1$ & & & & \\
\hline 0.702 & 0.687 & 0.452 & 0.282 & 0.078 \\
0.687 & 0.488 & 0.348 & 0.215 & 0.060 \\
0.452 & 0.348 & 0.253 & 0.149 & 0.042 \\
0.282 & 0.215 & 0.149 & 0.095 & 0.027 \\
0.078 & 0.060 & 0.042 & 0.027 & 0.007 \\
\hline (b) $-5 \%$ error at position $i=13$ & & & & \\
\hline 0.838 & 0.642 & 0.452 & 0.280 & 0.078 \\
0.642 & 0.487 & 0.380 & 0.212 & 0.061 \\
0.452 & 0.380 & 0.115 & 0.183 & 0.037 \\
0.280 & 0.212 & 0.183 & 0.092 & 0.028 \\
0.078 & 0.061 & 0.037 & 0.027 & 0.007 \\
\hline
\end{tabular}

verse by the direct method for case (i), when the probe output differs by $5 \%$ from the correct value at the probe position $i=1$ or $i=13$. The error brings differences of $31 \%$ and $54 \%$ at subareas $j=1$ and $j=13$, respectively, much larger than $5 \%$. Moreover, a difference of over $10 \%$ occurs at the neighboring subareas. In the same way, Tables 10 and 11 give the reproduced distribution with the probe outputs including $5 \%$ error at the position $i=13$ in case (ii) and at $i=12$ in case (iii), respectively. These tables reveal that the effect of the measurement error is much lower for local charging.

For case (iv), Table 12 gives the charge distributions calculated when the probe output has an error of $5 \%$ at a position $i=1$ or $i=13$. It can be seen that compared with the values in Table $8 \mathrm{a}$, the inverse calculation magnifies the error at or near the corresponding subarea. In particular, the relative difference at subarea $j=13$ is fifty times larger than without the assumed measurement error. Table 13 shows similar results for cases (i) and (iv) when the probe output has bipolar errors, that is, $-1 \%$ error at the positions of odd number and $1 \%$ error at the positions of even number. It can be seen that these errors of only $1 \%$ give rise to a relative difference of about $20 \%$ in all subareas for case (i). In case (iv) of the continuous distribution, 
Table 13. Effect of alternate positive and negative errors of $\pm 1 \%$ in probe output. (a) distribution for case (i) (in $\mathrm{pC}$ ); (b) distribution for case (iv) (in pC).

\begin{tabular}{ccccc}
\hline (a) distribution for case (i) & & & & \\
\hline 0.88 & 1.17 & 0.82 & 1.17 & 0.88 \\
1.17 & 0.79 & 1.22 & 0.79 & 1.17 \\
0.82 & 1.22 & 0.77 & 1.22 & 0.82 \\
1.17 & 0.79 & 1.22 & 0.79 & 1.17 \\
0.88 & 1.17 & 0.82 & 1.17 & 0.88 \\
\hline (b) distribution for case (iv) & & & & \\
\hline 0.789 & 0.702 & 0.401 & 0.322 & 0.052 \\
0.702 & 0.415 & 0.412 & 0.162 & 0.092 \\
0.402 & 0.412 & 0.192 & 0.197 & 0.010 \\
0.322 & 0.162 & 0.197 & 0.054 & 0.053 \\
0.052 & 0.092 & 0.010 & 0.053 & 0.012 \\
\hline
\end{tabular}

the charge distribution again shows large differences except for $j=1$ compared with the values in Table $8 \mathrm{a}$. It is very difficult in practice to measure probe outputs within an accuracy of $1 \%$.

\subsection{REDUCTION OF MATRIX CONDITION NUMBER}

As explained above, the measurement error has a large influence on the reproduced charge distribution. The condition number of a matrix is defined as the ratio of the largest singular value of the matrix to the smallest non-zero one. As well known in matrix calculations, the condition number should be as low as possible in order that the measurement errors have less effect on the result of the inverse calculation. A diagonal unit matrix has the smallest condition number equal to unity, which corresponds to the best condition for the inverse calculation. The smaller the condition number, the more reliable is the inverse calculation. There is a possibility of reducing the enlarging effect of measurement errors by diminishing the condition number of the corresponding matrix. Reducing the condition number has nothing to do with the right-hand side input vector in equation (2), which is used as an input in the inverse calculation. The condition number becomes smaller if we set to zero any singular value in the matrix much smaller than the largest one, which may lead to a reduction of the effect of measurement errors. However, the resulting matrix is no longer identical to the original one, so that the reproduced distribution may also be different from the one to be measured. There remains a problem as to what extent smaller singular values should be set to zero.

We have reduced the condition number from 19.4 to 10.7 in this way and applied it for the cases with the bipolar errors as given in Table 13. Table 14 shows the distribution reproduced by applying the matrix so obtained in the cases corresponding to Table 13. Comparison with Table 13 indicates that the reduction of the condition number has suppressed the effect of measurement errors, resulting in a better reproduction of the charge distribu-
Table 14. Effect of reducing the condition number. (a) distribution for case (i) (in pC); (b) distribution for case (iv) (in pC).

\begin{tabular}{ccccc}
\hline (a) distribution for case (i) & & & & \\
\hline 0.98 & 1.01 & 0.98 & 1.01 & 0.98 \\
1.01 & 1.03 & 1.00 & 1.03 & 1.01 \\
0.98 & 1.00 & 0.97 & 1.00 & 0.98 \\
1.01 & 1.03 & 1.00 & 1.03 & 1.01 \\
0.98 & 1.01 & 0.98 & 1.01 & 0.98 \\
\hline (b) distribution for case (iv) & & & & \\
\hline 0.818 & 0.654 & 0.451 & 0.276 & 0.079 \\
0.654 & 0.488 & 0.345 & 0.224 & 0.053 \\
0.451 & 0.346 & 0.242 & 0.152 & 0.041 \\
0.276 & 0.223 & 0.152 & 0.091 & 0.029 \\
0.079 & 0.053 & 0.041 & 0.029 & 0.003 \\
\hline
\end{tabular}

tions. However, the reduction of the condition number has not always given better results in our analysis. The matrix with the reduced condition number has brought about larger differences in cases (ii) and (iii) than the original one.

\section{CONCLUSIONS}

$\mathrm{T}$ HE measurement of surface charge has not yet been performed quantitatively for any practical high voltage supports. We have numerically analyzed a measurement system with a practical probe for thick specimens by applying the highly efficient surface charge method developed in our laboratory. The principal results are summarized as follows.

1. The calculated probe output varies significantly with the measuring position of the probe even if the solid surface is charged uniformly at a constant density. This is due to the effect of the surrounding charge not directly beneath the sensor, which is more serious in the arrangement with a thick specimen than with a flat slab.

2. We have compared the matrix components computed by the two numerical algorithms called the direct method and the $\lambda$-function method, and have achieved differences of the diagonal components by the two methods which are below $1 \%$.

3. We have used the calculated probe outputs as the inputs to the inverse calculation. The direct method reproduces the constant charge densitics with a largest error of $1.3 \%$, smaller than the $\lambda$-function method. In the case of a continuously changing charge distribution, the $\lambda$ function method gives better results.

4. Measurement errors, or errors in probe outputs, may cause much larger errors in the reconstructed charge distribution. The charge distribution is assumed to be constant and change smoothly. The reconstruction by the probe outputs with measurement errors of only $\pm 1 \%$ results in quite different charge distributions with differences of over $20 \%$ in some subareas. On the other hand, the measurement error is not enlarged so much when the surface is charged locally. 
IEEE Transactions on Dielectrics and Electrical Insulation

5. The reduction of the condition number of the matrix lessens the effect of output errors in some cases, which improves the reconstruction of the charge distribution. In particular, when the whole of the solid side is charged uniformly, we have successfully reconstructed the assumed distribution with the largest difference of $3 \%$ from outputs including measurement errors of $\pm 1 \%$.

\section{APPENDIX \\ 7.1 FIELD CALCULATION}

The numerical field calculation for Figure 1 needs a very fine division of the surfaces and boundaries, much finer than shown in Figure 3. We apply the following novel techniques which have been developed for the surface charge method in our laboratory.

(a) The solid dielectric in a cubic shape can be simulated accurately with only plane elements. The electrostatic probe having curved surfaces is represented by both curved and planar elements. The triangular curved surface element is expressed by a third-order shape function with nine degrees of freedom, while a quadrangular one is simulated by a third-order serendipity function with twelve degrees of freedom.

(b) Each subdivided element has a second-order charge density distribution with six degrees of freedom for a triangular element and eight degrees of freedom for a serendipity-type quadrangular one. This application of higher-order representation functions improves the accuracy of the field calculation. However, the important point to note is that the higher-order charge density representation often needs nodes at the edges of the solid. The boundary condition on a dielectric surface, the continuity of the normal component of flux density, cannot be defined at the edges where the normal direction is indefinite. Consequently, we apply non-conforming elements at the edges of the solid. This technique permits discontinu- ity of the charge density between neighboring elements, thus making it possible to use the higher-order function for charge density representation. We also apply non-conforming elements at the boundary of two subareas since a discontinuous charge distribution is often assumed between subareas.

\section{REFERENCES}

[1] H. Specht, "Oberflächenladungen bei Rotations-symmetrischen Isolierstoffkörpern", ETZ-A, Vol. 97, pp. 474-476, 1976.

[2] T. Takuma, M. Yashima and T. Kawamoto, "Principle of Surface Charge Measurement for Thick Insulating Specimens", IEEE Trans. DEI, Vol. 5, pp. 497-504, 1998.

[3] K. Nakanishi, A. Yoshioka, Y. Arahata, Y. Shibuya, "Surface Charging on Epoxy Spacer at DC Stress in Compressed $\mathrm{SF}_{6}$ Gas", IEEE Trans. PAS, Vol. 120, pp. 3919-3926, 1983.

[4] H. Ootera, K. Nakanishi, Y. Shibuya, Y. Arahata and T. Nitta, "Measurement of Charge Accumulation on Conical Spacer for 500 kV DC GIS", Gaseous Dielectrics IV, 1984, pp. 443-451.

[5] H. Ootera and K. Nakanishi, "Analytical Method for Evaluating Surface Charge Distribution on a Dielectric from Capacitive Probe Measurement - Application to a Cone-Type Spacer in \pm 500 kV DC-GIS", IEEE Trans. Power Delivery, Vol. 3, pp. $165-172,1988$.

[6] T. Takuma and S. Hamada, "Analysis of Measuring Methods for Accumulated Surface Charge", ELEKTRIE, Vol. 53, 1999, pp. $397-400$

[7] A. Tatematsu, S. Hamada, T. Takuma, "Calculation of the Coefficient Matrix between Surface Charge and Probe Response for Surface Charge Measurement", ACED-2000 (10th Asian Conference on Electrical Discharge), Kyoto, Japan, paper F-613, 2000.

[8] M. Yashima, H. Fujinami and T. Takuma, "Mcasurement of Accumulated Charge on Dielectric Surfaces with an Electrostatic Probe", Gaseous Dielectrics V, pp. 242-247, 1987.

[9] A. Pedersen, "On the Electrostatics of Probe Measurements of Surface Charge Densities", Gaseous Dielectrics V, pp. 235-240, 1987.

[10] T.O. Rerup, G.C. Crichton and I.W. McAllister, "Using the $\lambda$ Function to Evaluate Probe Measurements of Charged Dielectric Surfaces", IEEE Trans. Dielectrics EI, Vol. 3, pp. 770-777, 1996.

Manuscript received on 17 June 2001, in final form 4 February 2002. 